\title{
Self-reported halitosis and associated demographic and behavioral factors
}

\section{Fernanda Carpes MILANESI Bruno KAUER Tassiane Panta WAGNER Luciana Dondonis DAUDT Alex Nogueira HAAS}

Universidade Federal do Rio Grande do Sul - URFGS, Department of Periodontology, Faculty of Dentistry, Porto Alegre, RS, Brazil.

Declaration of Interests: The authors certify that they have no commercial or associative interest that represents a conflict of interest in connection with the manuscript.
Abstract: Halitosis is still poorly studied in young adults. The aim of this study was to evaluate the occurrence of self-reported halitosis and associate it with demographic and behavioral factors in young adult dental students. This cross-sectional study was designed as a census of students enrolled in three initial and three final semesters of a dental course in a Brazilian public university. Of 284 eligible students, $257(90.5 \%)$ completed a self-administered questionnaire. Self-reported halitosis was the primary study outcome, and was assessed with the question "do you feel you have bad breath?". Data on age, gender, frequency of tooth brushing and interproximal cleaning, tongue cleaning, mouth rinse use and dry mouth were collected using the questionnaire, and were considered independent variables. Of the students surveyed, $26.5 \%$ reported as never, $51.7 \%$ as rarely, $21.4 \%$ as sometimes, and $0.4 \%$ as always feeling they had halitosis. Morning halitosis was reported by $90.6 \%$ of those who reported halitosis. In the final multiple model, last semester students had a 55\% lower chance of reporting halitosis, compared with students from the first semesters [odds ratio (OR) 0.46; 95\%CI 0.24-0.89]. Women had a 2.57fold higher chance of reporting halitosis ( $\mathrm{OR}=2.57 ; 95 \% \mathrm{CI} 1.12-5.93)$. Dry mouth increased the chance of self-reported halitosis 3.95-fold, compared with absence of dry mouth (OR $=3.95 ; 95 \%$ CI 2.03-7.68). It can be concluded that self-reports of halitosis were low among dental students, but may represent an important complaint. Gender, dry mouth and level of college education of the dentist were factors significantly associated with self-reported halitosis.

Keywords: Halitosis; Students, Dental; Oral Hygiene; Risk Factors.

\section{Corresponding Author:}

Alex Nogueira Haas

E-mail: alexnhaas@gmail.com

DOI: 10.1590/1807-3107BOR-2016.vol30.0071

Submitted: May 20, 2015

Accepted for publication: Mar 07, 2016

Last revision: Apr 05, 2016

\section{Introduction}

Halitosis is characterized by a foul odor emanated from the oral cavity, and can affect people's quality of life and cause social restraints. ${ }^{1}$ Data on the prevalence of halitosis in different populations indicate great variability in the estimates. Nevertheless, the majority of studies suggest that moderate halitosis affects about one third of individuals, mentioning that there is very little evidence regarding the occurrence of halitosis in adolescents and young adults. Moreover, halitosis has been related to various factors, including physiological factors, such as biofilm accumulation in the tongue, going without eating for prolonged whereas severe halitosis affects less than $5 \%$ of the population. ${ }^{2}$ It's worth 
periods, ingestion of some types of foods/spices and tobacco use, ${ }^{3}$ as well as systemic ${ }^{4}$ and oral diseases, mainly periodontal diseases. ${ }^{5}$

One of the reasons for the high variability found in the prevalence estimates of halitosis is the existence of different halitosis diagnostic methods. The organoleptic measure has been regarded as the gold standard to determine halitosis. ${ }^{6}$ However, the organoleptic assessment is time-consuming and complex, requiring careful training and calibration of the examiner. Portable sulfide monitors that measure the levels of volatile sulfur compounds (VSC) emitted in the air have shown a good correlation with organoleptic scores, ${ }^{7,8}$ and have been used in clinical and epidemiologic studies. Self-reported halitosis has been used as a diagnostic tool, mostly in clinical practice and epidemiologic studies, since it reflects the patient's perspective regarding halitosis, and is of easy assessment. Self-reported halitosis studies have shown prevalence estimates between $22 \%$ and $40 \%$ in different populations. ${ }^{9,10,11,12}$ Nevertheless, it is known that self-reported halitosis tends to underestimate the real occurrence of this condition, mainly because of the difficulty of people to detect their own emanated odor or even because of constraints during the report. ${ }^{10}$

Studies indicate that the oral cavity is responsible for approximately $90 \%$ of halitosis cases. ${ }^{8,13,14}$ Thus, the dentist plays an essential role in diagnosing and treating halitosis. ${ }^{15}$ In this context, studies on the occurrence of halitosis have become important for dental professionals, including dental students, because they represent a positive model for the general population. However, dental students usually have a different pattern of health behavior and oral hygiene practices, ${ }^{16,17,18}$ taking this finding into consideration, they comprise an interesting population for the study of halitosis. Nevertheless, only one study that evaluated halitosis in dental students ${ }^{9}$ using self-report could be found in the literature. Another study evaluated only the concern regarding halitosis in dental students, ${ }^{19}$ and no study was found evaluating the occurrence of halitosis among dentists.
The objectives of this study were to evaluate the occurrence of self-reported halitosis in dental students from a southern Brazilian university, and to associate the occurrence of self-reported halitosis with demographic and behavioral factors.

\section{Methododology}

\section{Study design and sample}

The present study was a cross-sectional observational study. The target population comprised students from the School of Dentistry of the Universidade Federal do Rio Grande do Sul - UFRGS. At this school, the course takes over 5 years to complete and is organized into ten semesters. For the purpose of the present study, students from the first, second, third, eighth, ninth and tenth semesters were considered eligible. These semesters were chosen to allow comparisons between students in the beginning and in the end of the course.

The study comprised a census of students. Students enrolled in the abovementioned semesters were considered eligible for inclusion in the study. The study was conducted between August and December 2012, with 284 eligible students.

\section{Questionnaire}

A self-administered structured questionnaire with closed questions was applied in this study to assess sociodemographic and behavioral data. It was used in epidemiological studies conducted by our research group, and was tested and evaluated for its applicability. ${ }^{20,21}$ Additional questions about halitosis were included. The questionnaire was applied in the classrooms without any interference from the researchers. On average, it took 20 minutes to fill out the questionnaire.

\section{Response rate}

A strategy was used to increase the participation rate in the study. Students not present in the classroom when the study was conducted were contacted by telephone and scheduled to answer the questionnaire. Of all the eligible students, 257 responded the questionnaire, yielding a $90.5 \%$ response rate. 


\section{Dependent and independent variables}

The primary outcome of the present study was to assess self-reported halitosis through the question "do you feel you have bad breath?" The answers were recorded on a Likert scale (never, rarely, sometimes, always). The outcome variable for analysis of the factors associated with halitosis was dichotomized into yes (sometimes or always) and no (never or rarely). Secondary information about the period of self-perceived halitosis was also collected, in this case, by the question "If you feel you have bad breath, at what time during the day do you feel this?".

The independent variables studied were time attending the course, age, gender, frequency of tooth brushing, frequency of interproximal cleaning, tongue cleaning, use of mouth rinses and perception of dry mouth.

Age was categorized into 18-19 years old, 20-24 years old and over 25 years old. Tooth brushing frequency was dichotomized into at least twice a day and three or more times a day. Use of mouth rinse, frequency of interproximal cleaning and tongue cleaning were dichotomized into at least once a day and once or more times a day.

\section{Statistical analysis}

Descriptive data were expressed using frequency distribution. Univariable analyses were conducted with the chi-square test or Fisher's exact test, when appropriate, to evaluate the association between selfreported halitosis and independent variables. Uniand multivariable binary logistic regression models were applied to estimate the chance of self-reporting halitosis, according to independent variables expressing odds ratios (OR) and 95\% confidence intervals (95\%CI). Model building followed the purposeful approach proposed by Hosmer and Lemeshow. ${ }^{22}$ Independent variables showing associations with $p$-values $\leq 0.25$ in the univariable models were included in the multivariable model. Variables with $p$-values $<0.05$ were maintained in the final model. During model fitting, effect modification and interactions were evaluated. Effect modification was detected when one independent variable had a $25 \%$ changing effect in the regression coefficient of another variable. Effect modification and interactions were not found.

The analytical unit of this study was the individual, and the significance level was set at $5 \%$. Data were analyzed using Stata version 10 for Macintosh.

\section{Ethical aspects}

The present study was approved by the Ethics Committee of the UFRGS. An informed consent form was read to and signed by all participants. They were also informed that they had the right to refuse participation without any consequence to their course evaluation. No advice was given to individuals who reported halitosis, and the identification of participants remained concealed.

\section{Results}

Table 1 shows demographic and behavioral characteristics of the study participants. There was a similar distribution of participants in the initial and final semesters of the course. Most of the students were between 20-24 years old, were female, reported tooth brushing frequency of at least twice a day and interproximal cleaning of at least once a day. Approximately one third of the sample reported never using mouth rinses. More than $90 \%$ of the participants reported cleaning their tongue once or more times a day. Approximately $23 \%$ of the participants reported having dry mouth.

In relation to the occurrence of self-reported halitosis, $26.5 \%$ of the sample reported never noticing halitosis (Figure 1). More than half of the sample (51.7\%) said they rarely had halitosis. Higher frequencies of perceived halitosis, i.e., sometimes and always, were reported by $21.4 \%$ and $0.4 \%$, respectively. Only 9 (3.5\%) students reported that others alerted them about having halitosis. Among those who responded to feeling they had halitosis, $90.6 \%$ reported that they felt they had it in the morning (Figure 2). Only $6.6 \%$ and $2.8 \%$ of the students reported perceiving halitosis in the afternoon and evening, respectively.

The occurrence of dichotomized self-reported halitosis was $21.8 \%$ (Table 1). Approximately one third $(27.9 \%)$ of the students from the initial semesters reported having halitosis, compared with $14.9 \%$ of 
Table 1. Characteristics of the sample of the study.

\begin{tabular}{|c|c|c|c|c|c|c|c|}
\hline \multirow{3}{*}{ Variables } & \multirow{2}{*}{\multicolumn{2}{|c|}{ Whole sample }} & \multicolumn{5}{|c|}{ Self-reported halitosis } \\
\hline & & & \multicolumn{2}{|c|}{ Yes } & \multicolumn{2}{|c|}{ no } & \multirow[b]{2}{*}{$\mathrm{p}$} \\
\hline & $n$ & $\%$ & $n$ & $\%$ & $\mathrm{n}$ & $\%$ & \\
\hline \multicolumn{8}{|l|}{ Semester } \\
\hline Initial semesters $\left(7^{\text {st }}, 2^{\text {nd }}, 3^{\text {rd }}\right)$ & 136 & 52.9 & 38 & 27.9 & 98 & 72.1 & \multirow{2}{*}{$0.01^{5 *}$} \\
\hline Final semesters $\left(8^{\text {th }}, 9^{\text {th }}, 10^{\text {th }}\right)$ & 121 & 47.1 & 18 & 14.9 & 103 & 85.1 & \\
\hline \multicolumn{8}{|l|}{ Age } \\
\hline $18-19$ years & 52 & 20.2 & 12 & 23.1 & 40 & 76.9 & \multirow{3}{*}{$0.825^{*}$} \\
\hline $20-24$ years & 169 & 65.8 & 35 & 20.8 & 134 & 79.2 & \\
\hline$\geq 25$ years & 36 & 14.0 & 9 & 25.0 & 27 & 75.0 & \\
\hline \multicolumn{8}{|l|}{ Gender } \\
\hline Male & 73 & 28.4 & 8 & 10.9 & 65 & 89.0 & \multirow{2}{*}{$0.007^{\#}$} \\
\hline Female & 184 & 71.6 & 48 & 26.1 & 136 & 73.9 & \\
\hline \multicolumn{8}{|l|}{ Tooth brushing frequency } \\
\hline$\leq 2$ times/day & 201 & 78.2 & 48 & 23.9 & 153 & 76.1 & \multirow{2}{*}{$0.124^{\#}$} \\
\hline$\geq 3$ times/day & 56 & 21.8 & 8 & 14.3 & 48 & 85.7 & \\
\hline \multicolumn{8}{|c|}{ Frequency of interproximal cleaning } \\
\hline$<1$ time / day & 214 & 83.3 & 49 & 22.9 & 165 & 77.1 & \multirow{2}{*}{$0.337^{\#}$} \\
\hline$\geq 1$ time / day & 43 & 16.7 & 7 & 16.3 & 36 & 83.7 & \\
\hline \multicolumn{8}{|l|}{ Frequency of tongue cleaning } \\
\hline$<1$ time / day & 24 & 9.3 & 54 & 23.2 & 179 & 76.8 & \multirow{2}{*}{$0.120^{\#}$} \\
\hline$\geq 1$ time / day & 233 & 90.7 & 2 & 8.3 & 22 & 91.7 & \\
\hline \multicolumn{8}{|l|}{ Use of mouth rinse } \\
\hline Never & 81 & 31.5 & 23 & 28.4 & 58 & 71.6 & \multirow{3}{*}{$0.209^{*}$} \\
\hline$<1$ time / day & 90 & 35.0 & 16 & 17.8 & 74 & 82.2 & \\
\hline$\geq 1$ time / day & 86 & 33.5 & 17 & 19.8 & 69 & 80.2 & \\
\hline \multicolumn{8}{|l|}{ Dry mouth } \\
\hline No & 199 & 77.4 & 31 & 15.6 & 168 & 84.4 & \multirow{3}{*}{$<0.001^{\#}$} \\
\hline Yes & 58 & 22.6 & 25 & 43.1 & 33 & 56.9 & \\
\hline Total & 257 & 100.0 & 56 & 21.8 & 201 & 78.2 & \\
\hline
\end{tabular}

*Chi-square test; \#Fisher exact test.

those in the final semesters $(p=0.015)$. A significantly higher percentage of women $(26.1 \%)$ reported having halitosis compared with men (10.9\%). The occurrence of halitosis was significantly higher among students who reported dry mouth (43.1\%) compared with those who did not feel that they had it (15.6\%). There were no significant differences in the occurrence of halitosis between categories of age, tooth brushing frequency, interproximal cleaning, tongue cleaning and use of mouth rinse.

Table 2 presents uni- and multivariable logistic regression models of the association between demographic and behavioral variables with self-reported halitosis. In univariable models, the variables of semester, gender and dry mouth were significantly associated with self-reported halitosis. Mouth rinse use, tooth brushing frequency, interproximal cleaning and tongue cleaning presented $p<0.25$ in the univariable models, and were included in the multivariable model; however, they did not contribute to the final model, and were removed. In the final multivariable model, students from the final semesters had a 55\% lower chance of having halitosis compared to those from initial semesters. In addition, the female gender increased the odds of reporting halitosis approximately threefold, compared with the male gender. The presence of dry mouth showed a 3.95-fold increase in the odds of self-reported halitosis, in comparison with its absence. 


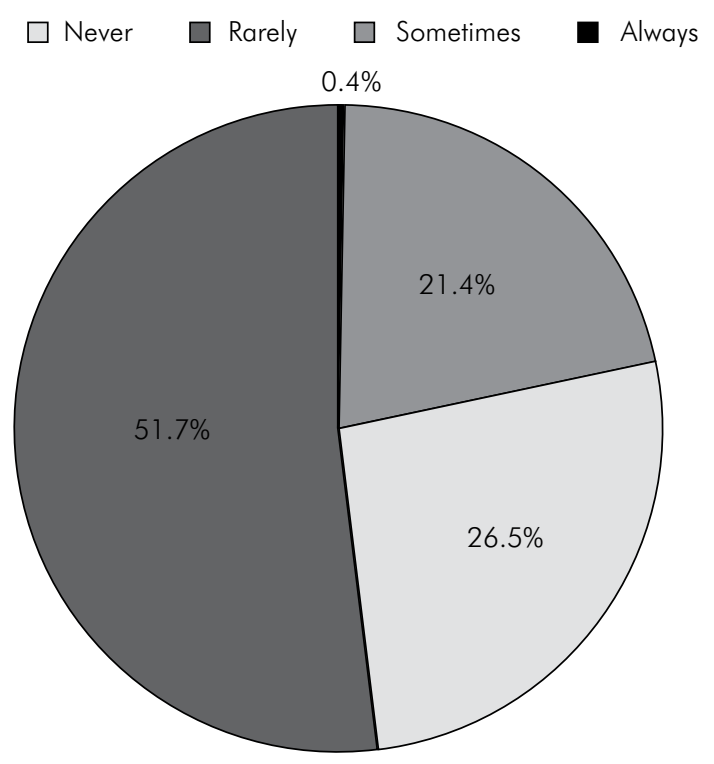

Figure 1. Occurrence of self-reported halitosis in the sample.
$\square$ Morning
$\square$ Afternoon
- Night

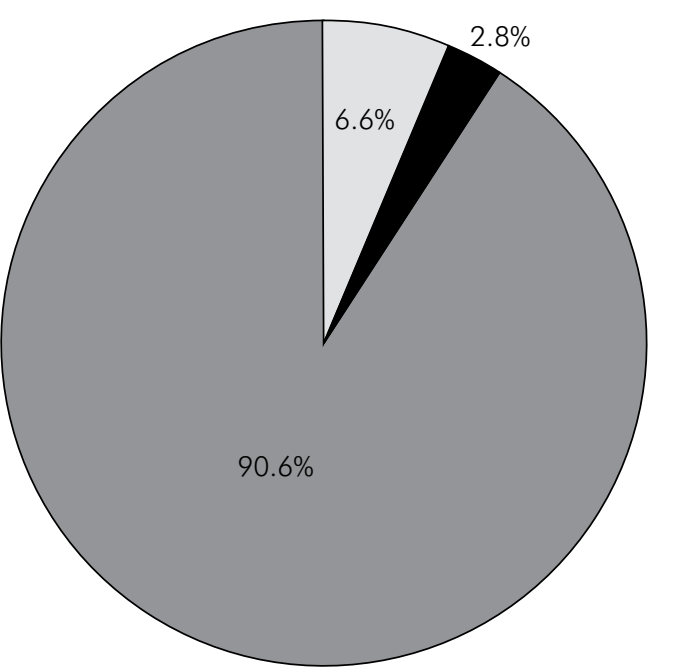

Figure 2. Periods of the day when self-reported halitosis occurred among students reporting halitosis.

Table 2. Uni- and multivariable logistic regression models of the association between demographic and behavioral variables with self-reported halitosis.

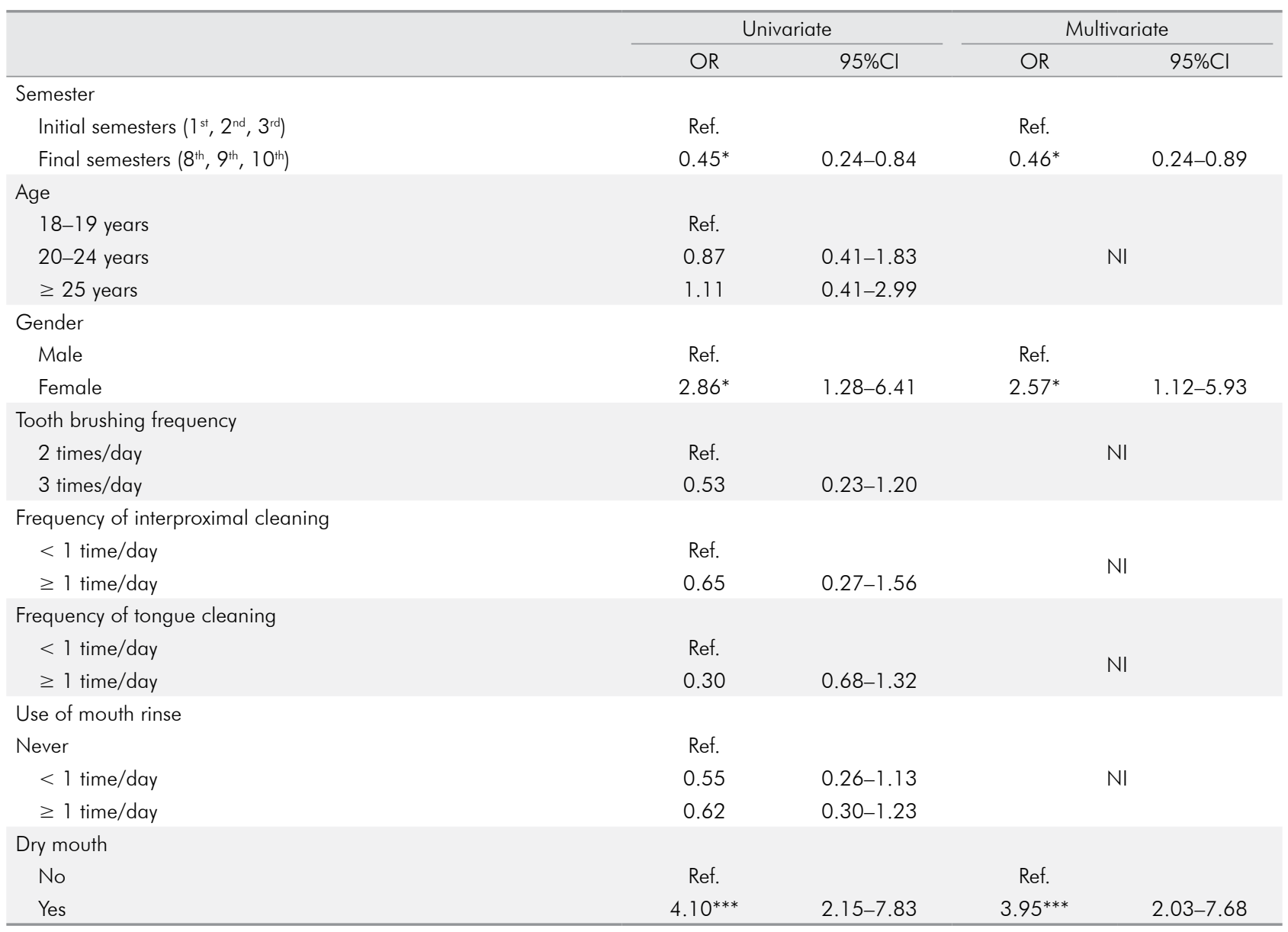

${ }^{*} \mathrm{p}<0.05 ;{ }^{* *} \mathrm{p}<0.01 ;{ }^{* * *} \mathrm{p}<0.001 ; \mathrm{NI}:$ not included in the model; Ref.: reference category. 


\section{Discussion}

In this study, a relatively low occurrence of self-reported halitosis was observed among dental students from a Brazilian dental school. Less than one third of the students reported having halitosis sometimes and always. Moreover, self-reported halitosis was significantly higher in beginner students, women and those who reported dry mouth. Age and habits related to oral hygiene were not associated with self-reported halitosis in this study.

The occurrence of self-reported halitosis was $21.8 \%$ in this study. Population-based studies showed variations in prevalence estimates of halitosis, ranging between $6 \%$ and $72 \% .2,23,24,25$ In comparison with other populations, the prevalence observed in this sample may be considered low. However, this prevalence of approximately 22\% may have an important impact, considering that the population studied is composed of dental students. Moreover, considering that the present sample reported preventive habits for halitosis, such as tongue cleaning, at a high rate, the observed prevalence of self-reported halitosis may also be considered of relevance.

Because halitosis was assessed by self-reporting, the occurrence observed in this study may be underestimated, since some participants could have found it difficult to detect or even report their own bad breath. Moreover, it must be acknowledged that organoleptic and volatile sulfur compound measurements are more reliable methods of detecting halitosis than self-reporting. ${ }^{10}$ Another aspect that may have contributed to a low occurrence of self-reported halitosis in this study is related to the fact that the sample was composed of dental students, whose oral hygiene and oral health patterns are different from those of the general population..$^{16,17,18}$ Since there are important associations between halitosis and periodontal disease, dental caries and poor oral hygiene, ${ }^{23}$ halitosis will consequently be lower in the absence of these conditions. Thus, it could be suggested that the self-reported halitosis by participants of this study is related to the dorsum of the tongue, or physiological halitosis, a fact supported by the high frequency of halitosis perception observed in the morning $(90.6 \%)$.

One previous study that evaluated halitosis in dental students also used self-reported perceptions of bad breath. ${ }^{9}$ The self-reported halitosis in this study was lower (21\%) than that found in the study carried out with Saudi
Arabian students (38\%), by Almas et al. ${ }^{9}$ They found a higher occurrence of self-reported halitosis in men than in women. In both studies, the majority of students reported feeling they had halitosis in the morning. However, direct comparisons should be made with caution since different methodologies were applied. Furthermore, the associations between halitosis and behavioral variables were adjusted for important factors through multivariable analysis only in the present study.

Self-reported halitosis was not associated with oral hygiene habits in this study. This may also be related to the better oral hygiene patterns seen among dental student ${ }^{16,17,18}$ and to the low variability observed in self-reported oral hygiene habits presented by the present sample, making it difficult to find significant associations. Furthermore, it is noteworthy that over $90 \%$ of the participants reported cleaning their tongue at least once a day, a habit that can have a significant impact in reducing halitosis, since the tongue plays an important role in halitosis, mainly in individuals with good oral health. ${ }^{24}$

The occurrence of self-reported halitosis was higher in initial semester students than in final semester students. The chance of detecting self-reported halitosis was 55\% lower in the students from the last three semesters than in those from the first three semesters. One explanation for this relationship is that more advanced students gained greater knowledge and may be able to better detect and prevent the presence of halitosis.

The occurrence of self-reported halitosis was $10 \%$ among men and $26 \%$ among women, representing threefold greater odds of females reporting halitosis. This difference may be attributed to the fact that women show better self-perception of their health conditions than men. ${ }^{26,27}$ Moreover, the hormonal oscillations related to the menstrual cycle, and the changes associated to salivary flow and protein concentration occurring in this period may change VSC concentration and, consequently, the presence of halitosis. ${ }^{23}$

Pseudo-halitosis is characterized by the absence of clinical determination of halitosis, but there is a clear complaint about it by the individual. ${ }^{2,28}$ This should be contextualized in the present study. One limitation of self-reported halitosis is the possible overestimation of the prevalence rates by pseudo-halitosis cases in the 
sample studied. ${ }^{28}$ It is uncertain whether this effect may be excluded in this study; however, taking into consideration the relatively low occurrence of halitosis in this study, it is probable that this effect was low. Another important aspect is that women seem to show a higher occurrence of pseudo-halitosis; ${ }^{8}$ therefore, the occurrence observed may be overestimated in this gender.

In this study, reporting dry mouth represented approximately fourfold higher odds of self-reported halitosis in dental students. Dry mouth has been suggested as a risk factor for halitosis, although it should be borne in mind that the number of studies in the dental area remains limited. ${ }^{29,30}$ Salivary flow reduction has also been associated with halitosis by facilitating protein degradation in the oral cavity, and consequently increasing production of sulfur compounds.

Among the limitations of the present study is the absence of a clinical examination to assess the students' oral health status. In addition, there was no information about self-reported measures taken against periodontal disease. The fact that halitosis was assessed by self-report may be considered a limitation by some researchers. Nevertheless, many studies have shown a positive correlation between an individual's self-perception about his oral health and his real condition, considering that self-perception is a predictor of oral status and may even be used as a therapeutic planning tool. ${ }^{31,32}$ Moreover, from a clinical perspective, individual-centered outcomes have been more frequently used in the dental literature, and self-reported halitosis fits this scenario well. The strengths of this study may also include the sample size and the high response rate, which allowed applying multivariable analytical models that control confounding factors. Furthermore, according to the profile of the studied sample, it can be inferred that this study refers to physiological halitosis, and, thus, the data showed here could be extrapolated to individuals with similar demographic characteristics.

\section{Conclusions}

Self-reported halitosis had a low occurrence among this study sample of Brazilian dental students, but may represent an important complaint among dental students. The female gender, self-reported oral dryness, and students attending their early college years were factors significantly associated with self-reported halitosis.

\section{References}

1. Zaitsu T, Ueno M, Shinada K, Wright FA, Kawaguchi Y. Social anxiety disorder in genuine halitosis patients. Health Qual Life Outcomes. 2011 Nov 3;9:94. doi:10.1186/1477-7525-9-94

2. Rösing CK, Loesche W. Halitosis: an overview of epidemiology, etiology and clinical management. Braz Oral Res. 2011;25(5):466-71. doi:10.1590/S1806-83242011000500015

3. Suarez F, Springfield J, Furne J, Levitt M. Differentiation of mouth versus gut as site of origin of odoriferous breath gases after garlic ingestion. Am J Physiol. 1999;276(2 1):G425-30.

4. Tangerman A. Halitosis in medicine: a review. Int Dent J. 2002;52(Supp 5 Pt 1):201-6. doi:10.1002/j.1875-595X.2002.tb00925.x

5. Bosy A, Kulkarni GV, Rosenberg M, McCulloch CA. Relationship of oral malodor to periodontitis: evidence of independence in discrete subpopulations. J Periodontol. 1994;65(1):37-46. doi:10.1902/jop.1994.65.1.37

6. Yaegaki K. Advances in breath odor research: re-evaluation and newly-arising sciences. J Breath Res [Internet]. 2012 [cited 2016 Apr 4];6(1):010201.
Available from: http://iopscience.iop.org/ article/10.1088/1752-7155/6/1/010201/meta;jsession id=CF218BCE3407B8B98C3806BCC90229A9.c3\#top. doi:10.1088/1752-7155/6/1/010201

7. Brunner F, Kurmann M, Filippi A. The correlation of organoleptic and instrumental halitosis measurements. Schweiz Monatsschr Zahnmed. 2010;120(5):402-8.

8. Quirynen M, Dadamio J, Van den Velde S, De Smit M, Dekeyser C, Van Tornout M, et al. Characteristics of 2000 patients who visited a halitosis clinic. J Clin Periodontol. 2009;36(11):970-5. doi:10.1111/j.1600-051X.2009.01478.x

9. Almas K, Al-Hawish A, Al-Khamis W. Oral hygiene practices, smoking habit, and self-perceived oral malodor among dental students. J Contemp Dent Pract. 2003;4(4):77-90.

10. Bornstein MM, Kislig K, Hoti BB, Seemann R, Lussi A. Prevalence of halitosis in the population of the city of Bern, Switzerland: a study comparing self-reported and clinical data. Eur J Oral Sci. 2009;117(3):261-7. doi:10.1111/j.1600-0722.2009.00630.x

11. Frexinos J, Denis P, Allemand H, Allouche S, Los F, Bonnelye G. [Descriptive study of digestive functional symptoms in the French general 
population]. Gastroenterol Clin Biol. 1998;22(10):785-91. doi:GCB-11-1998-22-10-0399-8320-101019-ART94

12. Loesche WJ, Grossman N, Dominguez L, Schork MA. Oral malodour in the elderly. In: van Steenberghe D, Rosenberg M, editors. Bad breath: a multidisciplinary approach. Leuven: Leuven University Press; 1996. p. 181-94.

13. Delanghe G, Ghyselen J, Feenstra L, van Steenberghe D. Experiences of a Belgian multidisciplinary breath odour clinic. Acta Otorhinolaryngol Belg. 1997;51(1):43-8.

14. McNamara TF, Alexander JF, Lee M. The role of microorganisms in the production of oral malodor. Oral Surg Oral Med Oral Pathol. 1972;34(1):41-8. doi:10.1016/0030-4220(72)90271-X

15. Seemann R, Conceição MD, Filippi A, Greenman J, Lenton $\mathrm{P}$, Nachnani S, et al. Halitosis management by the general dental practitioner-results of an international consensus workshop. J Breath Res [Internet]. 2014 [cited 2016 Apr 5];8(1):017101. Available from: http:// iopscience.iop.org/article/10.1088/1752-7155/8/1/017101/ meta. doi:10.1088/1752-7155/8/1/017101

16. Chiappe V, Gómez M, Pedreira P, Galeano A, Grinfeld A, Viale J, et al. Longitudinal study of periodontal condition in students of the Dental School the University of Buenos Aires Argentina. Acta Odontol Latinoa. 1997;10(2):117-32.

17. Matas F, Sentís J, Mendieta C. Ten-year longitudinal study of gingival recession in dentists. J Clin Periodontol. 2011;38(12):1091-8. doi:10.1111/j.1600-051X.2011.01799.x

18. Orth CC, Reichert MR, Leitune VB, Haas AN, Rösing CK. Compostos sulforados voláteis, placa dental e gengivite em estudantes de odontologia da Universidade Federal do Rio Grande do Sul. Rev Fac Odontol P Alegre. 2003;44(2):26-30.

19. Komabayashi T, Kwan SY, Hu DY, Kajiwara K, Sasahara H, Kawamura M. A comparative study of oral health attitudes and behaviour using the Hiroshima University - Dental Behavioural Inventory (HU-DBI) between dental students in Britain and China. J Oral Sci. 2005;47(1):1-7. doi:10.2334/josnusd.47.1

20. Susin C, Dalla Vecchia CF, Oppermann RV, Haugejorden $\mathrm{O}$, Albandar JM. Periodontal attachment loss in an urban population of Brazilian adults: effect of demographic, behavioral, and environmental risk indicators. J Periodontol. 2004;75(7):1033-41. doi:10.1902/jop.2004.75.7.1033

21. Haas AN, Gaio EJ, Oppermann RV, Rösing CK, Albandar JM, Susin C. Pattern and rate of progression of periodontal attachment loss in an urban population of South Brazil: a 5-years population-based prospective study. J Clin Periodontol. 2012;39(1):1-9. doi:10.1111/j.1600-051X.2011.01818.x
22. Hosmer DW, Lemeshow S. Applied Logistic Regression. 2nd ed. [place unknown]: John Wiley \& Sons, Inc.; 2000. doi:10.1002/0471722146

23. Liu XN, Shinada K, Chen XC, Zhang BX, Yaegaki K, Kawaguchi Y. Oral malodor-related parameters in the Chinese general population. J Clin Periodontol. 2006;33(1):31-6. doi:10.1111/j.1600-051X.2005.00862.x

24. Miyazaki H, Sakao S, Katoh Y, Takehara T. Correlation between volatile sulphur compounds and certain oral health measurements in the general population. J Periodontol. 1995;66(8):679-84. doi:10.1902/jop.1995.66.8.679

25. Cortelli JR, Barbosa MD, Westphal MA. Halitosis: a review of associated factors and therapeutic approach. Braz Oral Res. 2008;22(Suppl 1):44-54. doi:10.1590/S1806-83242008000500007

26. Ekbäck G, Astrøm AN, Klock K, Ordell S, Unell L. Self-perceived oral health among 19-year-olds in two Swedish counties. Swed Dent J. 2008;32(2):83-93.

27. Fernandez E, Schiaffino A, Rajmil L, Badia X, Segura A. Gender inequalities in health and health care services use in Catalonia (Spain). J Epidemiol Community Health. 1999;53(4):218-22. doi:10.1136/jech.53.4.218

28. Yaegaki K, Coil JM. Genuine halitosis, pseudo-halitosis, and halitophobia: classification, diagnosis, and treatment. Compend Contin Educ Dent. 2000;21(10A):880-6, 888-9; quiz 890.

29. Albuquerque DF, Tolentino ES, Amado FM, Arakawa C, Chinellato LEM. Evaluation of halitosis and sialometry in patients submitted to head and neck radiotherapy. Med Oral Patol Oral Cir Bucal. 2010;15(6):e850-4. doi:10.4317/medoral.15.e850

30. Koshimune S, Awano S, Gohara K, Kurihara E, Ansai T, Takehara T. Low salivary flow and volatile sulfur compounds in mouth air. Oral Surg Oral Med Oral Pathol Oral Radiol Endod. 2003;96(1):38-41. doi:10.1016/S1079-2104(03)00162-8

31. David J, Astrøm AN, Wang NJ. Prevalence and correlates of self-reported state of teeth among schoolchildren in Kerala, India. BMC Oral Health [Internet]. 2006 [cited 2016 Apr 4];6:10. Available from: http://bmcoralhealth.biomedcentral.com/ articles/10.1186/1472-6831-6-10. doi:10.1186/1472-6831-6-10

32. Iwanicka-Grzegorek E, Michalik J, Kepa J, Wierzbicka M, Aleksinski M, Pierzynowska E. Subjective patients' opinion and evaluation of halitosis using halimeter and organoleptic scores. Oral Dis. 2005;11(Suppl s1):86-8. doi:10.1111/j.1601-0825.2005.01101.x 